Ann. Zootech., I974, 23 (4), 459-480.

\title{
EFFETS DE LA VITESSE ET DU RAPPORT DE PULSATION SUR LES CARACTÉRISTIQUES DE TRAITE A LA MACHINE DES BREBIS PREALPES DU SUD
}

\author{
J. LABUSSIÈRE, J. LE DU, Madeleine DOUAIRE \\ et J.-F. COMBAUD
}

avec la collaboration technique de P. Petrequin et B. Mirman

Laboratoire de Recherches sur la Physiologie et la Technologie de la Traite,

Centre de Recherches de Rennes, I. N.R. A.

35042 Rennes Cedex

\section{RÉSUMÉ}

Ce travail a pour but de comparer l'efficacité de 3 vitesses de pulsations (6o, I20 et I $80 \mathrm{p} / \mathrm{mn})$ et de 3 rapports succion massage (33 p. I0o, 50 p. I 00,75 p. Ioo) sur la production laitière et les caractéristiques de traite des brebis Préalpes $d u$ Sud.

L’organisation générale de l'expérience est schématisée à la figure 6 . Pendant une période pré-expérimentale de 28 jours qui commence après un bref allaitement de 48 heures, tous les animaux sont soumis aux mêmes conditions de traite mécanique : les pulsateurs sont alors réglés à $50 \mathrm{p}$. $100-\mathrm{I}$ Bo $\mathrm{p} / \mathrm{mn}$.

Après ces 4 premières semaines les 207 brebis (dont les caractéristiques sont résumées au tableau I) sont réparties dans 9 lots équilibrés. Chacun d'entre eux va recevoir pendant une période expérimentale de 98 jours l'un des 9 traitements combinant les 3 rapports et les 3 vitesses de pul-

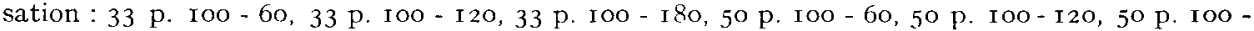
I 80,75 p. IOO-60, 75 p. IOO-I 20,75 p. IOO - I 80 (fig. f).

Il apparaît au tableau 4 que :

- Ces 9 combinaisons ne semblent pas affecter la production de matières grasses ainsi que les volumes de lait total, de lait extrait par la machine avant l'intervention du trayeur et d'égouttage machine.

- L'égouttage manuel et l'égouttage total diffèrent significativement d'un lot à l'autre et le test de Duncan (tabl. 8 et 9) révèle que la vitesse la plus rapide ( $80 \mathrm{p} / \mathrm{mn}$ ) associée à des rapports succion massage élevés (50 p. Ioo ou $75 \mathrm{p}$. 100) conduit pour ces 2 critères à des volumes plus importants que ceux obtenus avec les associations $33 \mathrm{p}$. roo- 60 et $33 \mathrm{p}$. Ioo - I 20 qui peuvent donc être considérées comme les plus favorables.

L'analyse de variance présentée au tableau 5 ne permet pas de déceler d'intéractions entre la vitesse et le rapport de pulsation.

En revanche :

- La réduction de la vitesse entraîne une diminution significative de l'égouttage machine et hautement significative de l'égouttage manuel et total (tabl. 5). 
Statistiquement, on ne relève pas de différence entre $60 \mathrm{p} / \mathrm{mn}$ et $\mathbf{1} 20 \mathrm{p} / \mathrm{mn}$ alors que le rythme de $180 \mathrm{p} / \mathrm{mn}$ entraîne un égouttage nettement plus important (tabl. 6).

Les modifications du rapport n'affectent que l'égouttage manuel $(\mathrm{P}<0,05$ : tabl. 5). Le volume de repasse obtenu à 33 p. Ioo est significativement plus faible que celui recueilli avec les traitements 50 p. Ioo et 75 p. Ioo qui ne semblent pas différer entre eux (tabl. 7).

Les effets bénéfiques de la réduction du rapport et de la vitesse de pulsation semblent résulter de l'augmentation du temps dont dispose alors le manchon pour se fermer et décongestionner efficacement le trayon (fig. 9).

\section{INTRODUCTION}

Dès I923, deux français, C. Toulouse et A. Combes, envisagent de traire les brebis à la machine pour remplacer " la traite manuelle à la pincée, qui nécessite chaque jour, pour un troupeau moyen de 200 laitières, le travail de 5 personnes pendant quatre heures".

Toutefois, il faut attendre I932 pour que R. FLEURY, directeur de la Société des Caves et des Producteurs de Roquefort, propose, en collaboration avec la firme Alfa-Laval, un matériel adapté de façon satisfaisante à l'espèce ovine. Ce dernier diffère de celui utilisé pour les vaches par la taille et la forme de ses manchons trayeurs mais aussi par sa vitesse de pulsation très élevée pouvant atteinđre le rythme de I80 par minute. Malheureusement FI,EURY n'a jamais publié les résultats de ses essais et nous ne disposons pour les apprécier que des quelques enseignements rapportés par GAITIER (I943) lors de la rédaction d'une thèse de médecine vétérinaire. Depuis cette époque, cette machine, peu modifiée dans son principe, s'est lentement implantée en France, surtout à la suite de son adaptation dans la salle de traite en épi de type "Casse " proposée par Bosc (I963).

Sous des formes voisines et des appellations parfois différentes elle s'est également introduite à l'étranger, notamment en Israël (FINCI, I958), en Italie (CASU, I967) et plus récemment en Espagne.

Elle a enfin servi de référence et bien souvent de " modèle " pour les prototypes construits et essayés en Bulgarie (Dimov, ig63; Dimov, Tanev et ShaliCHEV, I966), en Hongrie (GAAL, I969) ou en Tchécoslovaquie (HoRAK, I964; Kolar, I966 et MASAR, I964, I968 $a, \operatorname{I9} 68 b, \operatorname{Ig} 68 c, \operatorname{Ig} 68 d, \mathbf{I} 972$ ).

Pourtant la traite mécanique des brebis pose encore actuellement de nombreux problèmes qui pourraient certainement être partiellement résolus en améliorant le fonctionnement du matériel traditionnel. C'est ainsi que dans la race Préalpes, nos propres travaux (LABUSSIÈRE et MARTINET, I964; LABUSSIÈRE et RICORDEAU, I970) ont permis de révéler l'existence de deux types de brebis laitières;

Io Celles faciles à traire à la machine qui fournissent successivement leur lait citernal puis leur alvéolaire, lait chassé des acini sous l'effet du réflexe neuro-endocrinien d'éjection (animaux à 2 émissions; Labussière, Mar'Tine'T et Dénamur, ig69).

$2^{\circ}$ Celles inaptes à la traite qui se différencient des précédentes par l'absence de ce réflexe, retenant ainsi une grande partie de leur lait et surtout de leurs matières grasses (jusqu'à 75 p. IOO : LABUssiÈrE, I969) dans les parties supérieures de la glande mammaire (animaux à une émission). 
Cette rétention se traduit par une réduction de la production laitière (environ 30 p. IOO, LABUSSI⿳亠े⿵冂卄 celle de la traite du dimanche soir (LABussière, Combaud et Petrequin, I974).

La " transformation " des brebis à une émission en brebis à deux émissions paraît donc souhaitable pour l'économie laitière ovine. Il n'est pas impossible, par exemple, qu'on puisse aboutir à ce résultat en réduisant la congestion des trayons et l'inconfort qui en résulte. Ce traumatisme généralement visible pendant les premiers jours de traite est très probablement dû à l'inefficience de la phase de massage que nous avons souvent constatée à $\mathbf{I} 80$ pulsations par minute. A ce rythme la pression atmosphérique s'installe en effet difficilement dans l'espace annulaire du gobelet et le manchon en caoutchouc n'a pas le temps de se " plier " complètement pour chasser efficacement le sang de 1'extrémité du trayon. A cet égard, on peut regretter que les caractéristiques de la pulsation et les facteurs qui en conditionnent l'efficacité n'aient pas donné lieu à davantage de recherches permettant de justifier (ou éventuellement de remettre en cause) les choix effectués par FLEURY il y a maintenant 40 ans.

Les quelques études conduites jusqu'à maintenant sont d'ailleurs peu convaincantes, car elles portent sur un nombre trop limité d'animaux ou sur des périodes expérimentales trop courtes: (Kolar, I966; SuCCI, CuTry et ENNE, I970 ; SuCCI, ENNE et CUTRY, I97I; ENNE, I972).

Il nous a donc paru important d'approfondir cette question afin de proposer rapidement un rapport et une vitesse de pulsation mieux appropriés à la décongestion des trayons.

Nous rapportons ci-après les résultats obtenus en ce domaine au cours d'une expérience dont 1'objet est la comparaison des effets de 3 rapports ( 33 p. Ioo, $5 \circ$ p. Ioo, $75 \mathrm{p}$. Iоo) et de 3 vitesses $(60 \mathrm{p} / \mathrm{mn}, \mathbf{1} 20 \mathrm{p} / \mathrm{mn}$, I $80 \mathrm{p} / \mathrm{mn})$

\section{MATÉRIEL ET MÉTHODES}

\section{A. - Protocole expérimental et constitution des lots}

Après l'agnelage provoqué par une injection intra-musculaire de $16 \mathrm{mg}$ de Dexamethasonc, les 207 brebis Pyéalpes du Sud utilisées pour cette expérience allaitent leur agneau pendant 2 journées en moyenne puis passent sans transition à la traite à la machine. Pendant une période préexpérimentale de 28 jours, tous les animaux sont traits dans les mêmes conditions et avec la même pulsation : vitesse $\mathrm{I} 80 \mathrm{p} / \mathrm{mn}$ et rapport de $50 \mathrm{p}$. Ioo.

A la fin de la $4^{\text {e }}$ semaine de cette période, les brebis sont réparties dans 9 lots équilibrés ( ${ }^{1}$ ) qui vont respectivement recevoir pendant une période expérimentale de 98 jours les 9 traitements indiqués au tableau $\mathrm{I}$, qui correspondent à la combinaison des 3 vitesses $(60 \mathrm{p} / \mathrm{mn}, 120 \mathrm{p} / \mathrm{mn}$, $\mathrm{I} 80 \mathrm{p} / \mathrm{mn}$ ) et des 3 rapports (33 p. I00, 50 p. I00, 75 p. 100).

\section{B. - Technique de traite}

Les gobelets sont posés sans préparation préalable de la glande mammaire. Lorsque le lait cesse de couler et avant de décrocher le faisceau trayeur, un égouttage machine est pratiqué en

(1) Ces lots sont équilibrés au regard des critères suivants : lait total (LT), lait d'égouttage total (LET), quantité de matières grasses (MG), caractère brebis à " une émission " ou à " deux émissions ". 
massant énergétiquement la mamelle. Enfin, un ouvrier spécialisé effectue un égouttage manuel ou " repasse ". La traite qui dure environ 2 heures a lieu 2 fois par jour: le matin à 6 heures, le soir à 16 heures. L'ordre de passage des lots est modifié tous les i 5 jours.

\section{TABLEAU I}

Caractéristiques de pulsation des différcnts lots et répartition des brebis dans chacun d'entre eux en fonction de leur numéro de lactation

\begin{tabular}{|c|c|c|c|c|c|c|c|c|c|c|c|}
\hline \multirow{4}{*}{$\begin{array}{l}\text { Caractéristiques } \\
\text { de la pulsation }\end{array}$} & \multirow[b]{3}{*}{$\begin{array}{l}\text { Vitesse de pulsation } \\
\text { (V) en } \mathrm{p} / \mathrm{mn}\end{array}$} & \multicolumn{9}{|c|}{ Numéro du lot } & \multirow{4}{*}{$\begin{array}{l}\text { Nbre } \\
\text { total } \\
\text { de } \\
\text { brebis }\end{array}$} \\
\hline & & \multirow{2}{*}{$\begin{array}{r}1 \\
- \\
601\end{array}$} & \multirow{2}{*}{$\begin{array}{c}2 \\
60\end{array}$} & \multirow{2}{*}{$\begin{array}{l}3 \\
-10 \\
\end{array}$} & \multirow{2}{*}{$\begin{array}{c}4 \\
-- \\
120\end{array}$} & \multirow{2}{*}{$\begin{array}{c}5 \\
120\end{array}$} & \multirow{2}{*}{$\begin{array}{c}6 \\
-\ldots \\
120\end{array}$} & \multirow{2}{*}{$\begin{array}{c}7 \\
180\end{array}$} & \multirow{2}{*}{$\begin{array}{c}8 \\
180\end{array}$} & \multirow{2}{*}{$\begin{array}{c}9 \\
180\end{array}$} & \\
\hline & & & & & & & & & & & \\
\hline & $\begin{array}{l}\text { Rapport de pulsation } \\
\text { (R) en p. } 100\end{array}$ & 33 & 50 & 75 & 33 & 50 & 75 & 33 & 50 & 75 & \\
\hline & 2e lactation & 11 & 9 & 12 & 10 & 10 & 11 & 8 & 13 & 16 & 100 \\
\hline Nombre de brebis & $3^{e}$ lactation & 7 & 5 & 5 & $t_{4}$ & 6 & 5 & 5 & 4 & 4 & 45 \\
\hline Répartition en & 'e lactation & ' & 7 & 4 & 6 & 5 & 4 & 8 & 6 & 3 & 47 \\
\hline fonction du no & 5e lactation & 1 & 2 & $z$ & 3 & 2 & 3 & 2 & $\ldots$ & - & 15 \\
\hline & Total & 23 & 23 & $2: 3$ & 23 & $2: 3$ & 23 & 23 & 23 & 23 & 207 \\
\hline
\end{tabular}

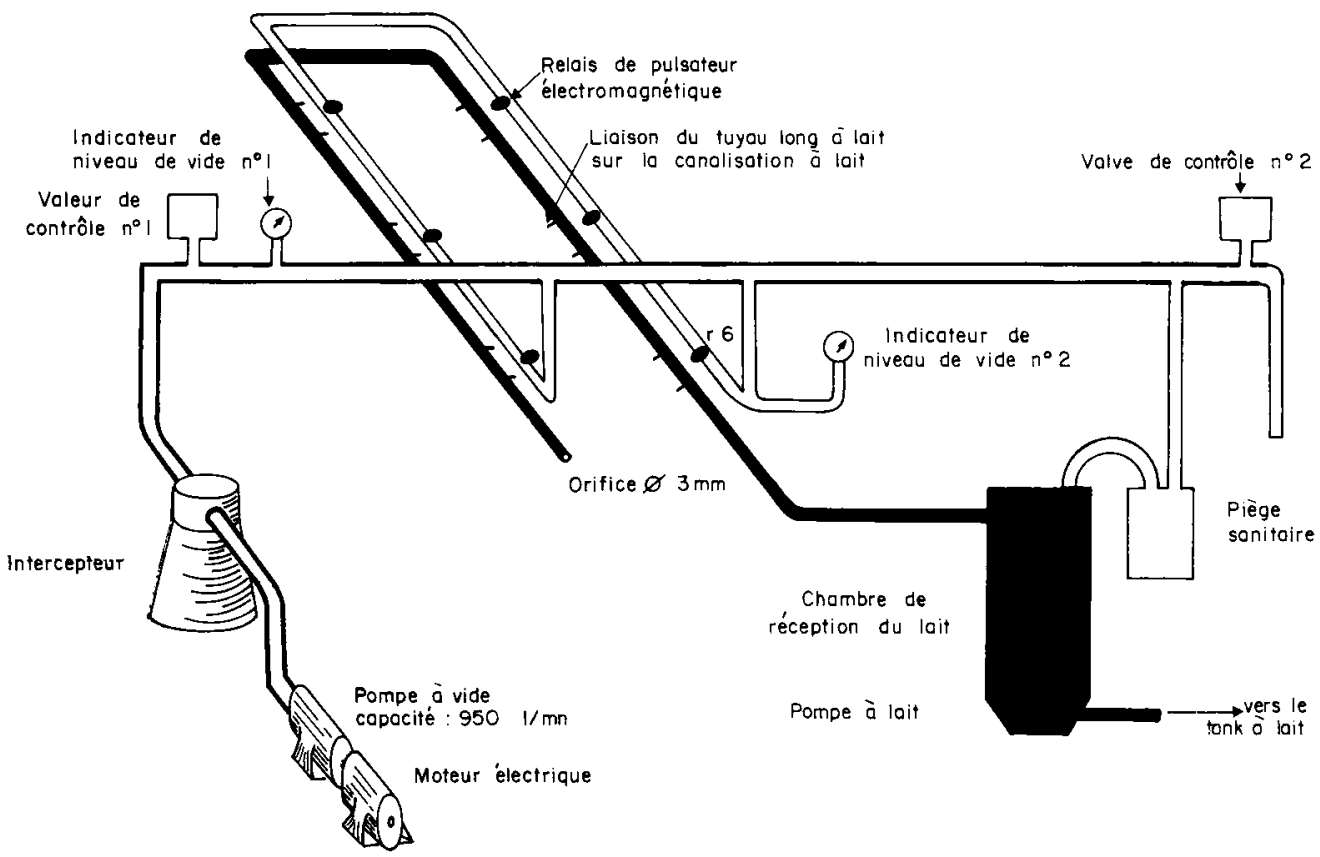

Frg. x. - Schéma de l'installation de traite

- canalisation à lait en pyrex $34 / 40$

- canalisation de vide : $-40 / 49$ de la pompe à vide à la valve de contrôle $n^{\circ} 2$

$-26 / 34$ autres parties de l'installation 


\section{C. - Matériel de traite}

\section{Caractéristiques générales de l'installation.}

La salle de traite Alfa-Laval, de type "Casse » (Bosc, I963), est décrite à la figure I.

Les caractéristiques de ses composants sont les suivantes :

a) I2 faisceaux trayeurs équipés de gobelets $n^{0} 26895$ (poids du gobelet seul : $144 \mathrm{~g} ; \varnothing$ intérieur : $30 \mathrm{~mm}$; hauteur r ro $\mathrm{mm}$ ) et les manchons $\mathrm{n}^{0} 25200$ (poids du manchon : $33 \mathrm{~g} ; \varnothing$ intérieur : I9 $\mathrm{mm}$ ).

b) I maître-pulsateur électromagnétique prototype permettant la sélection indépendante des vitesses et des rapports. Le bouton de sélection du rapport est un contacteur dont 3 positions distinctes correspondent aux rapports $33 \mathrm{p}$. Ioo, $50 \mathrm{p}$. Ioo et $75 \mathrm{p}$. Ioo. Les vitesses 60 , r 20 et I 80 pulsations par minute sont obtenues par positionnement du bouton de commande d'un potentiomètre devant des repères fixes d'un cadran. Il est à noter que ce potentiomètre, en offrant une possibilité de réglage continu de la vitesse et non un système de sélection par " tout ou rien ", introduit une certaine imprécision quant à la valeur exacte de la vitesse affichée.

c) 6 relais électromagnétiques $n^{\circ} 2624^{\circ}$, chaque relais étant relié à 2 faisceaux trayeurs.

d) 2 valves de contrôle du vide (ou régulateurs) - types à poids - référence $\mathrm{VV}_{4}{ }^{\circ}$, assurant un vide de $33 \mathrm{~cm}$ de mercure.

e) 2 indicateurs de niveau de vide (ou manomètres; type Bourdon).

f) Pompe à vide type VP 76 ; vitesse de rotation I 150 tours $/ \mathrm{mn}$; capacité en litres d'air atmosphérique extraits : $95^{\circ} 1 / \mathrm{mn}$ à $38 \mathrm{~cm} \mathrm{Hg}$ et I $1341 / \mathrm{mn}$ à $33 \mathrm{~cm} \mathrm{Hg}$.

g) Moteur électrique d'entraînement de la pompe à vide; fabrication russe; puissance $3 \mathrm{CV}$; vitesse de rotation : I $400 \mathrm{tr} / \mathrm{mn}$.

h) Lactoduc en verre pyrex situé sous le quai : $34 \mathrm{~mm} / 40 \mathrm{~mm}$.

i) Canalisation à vide :

- Entre la pompe et la valve de contrôle no $2: 40 \mathrm{~mm} / 49 \mathrm{~mm}$.

- Autres parties de l'installation, et notamment sous le quai : $26 \mathrm{~mm} / 34 \mathrm{~mm}$.

j) I 2 bocaux mesureurs montés entre la griffe et le lactoduc et permettant d'apprécier la production laitière avec une précision voisine de $10 \mathrm{ml}$.

\section{Contróle des débits d'air.}

Pour effectuer ces mesures, les manchons sont obstrués avec des bouchons et le débitmètre à orifices prend la place de la valve de contrôle de vide $\mathrm{n}^{\circ} 2$ mise hors service.

Les résultats exprimés en litre d'air atmosphérique par minute sont rapportés au tableau 2.

TABLEAU 2

Consommation d'air des éléments constitutifs de l'installation

\begin{tabular}{|c|c|c|c|c|c|c|}
\hline $\begin{array}{l}\text { Niveau de } \\
\text { vide en } \mathrm{cm} \\
\text { de } \mathrm{Hg}\end{array}$ & $\begin{array}{l}\text { Pompe à } \\
\text { vide seule }\end{array}$ & $\begin{array}{c}\text { Canalisation } \\
\text { à vide }\end{array}$ & Lactoduc & $\begin{array}{l}\text { Avec récipients } \\
\text { stockage } \\
\text { individuel }\end{array}$ & $\begin{array}{l}\text { Avec récipients } \\
\text { griffe et gobe- } \\
\text { lets bouchćs }\end{array}$ & $\begin{array}{c}\text { Avec récipients } \\
\text { griffe, gobelets, } \\
\text { pulsateurs en } \\
\text { marche }\end{array}$ \\
\hline 30 & 1200 & 1104 & 1076 & 1074 & 1024 & 820 \\
\hline $33^{*}$ & 1134 & 1016 & 1000 & 1000 & 958 & 748 \\
\hline 36 & 1030 & 938 & 900 & 900 & 856 & $6^{\prime}+0$ \\
\hline 38 & 986 & 870 & 846 & 846 & 800 & 600 \\
\hline \multicolumn{2}{|c|}{$\begin{array}{c}\text { Consommation au vide } \\
\text { de traite }\end{array}$} & 118 & 16 & 0 & 42 & 210 \\
\hline
\end{tabular}


3. Contrôle de la pulsation.

a) Définitions.

La pulsation est définie selon les recommandations de la Fédération internationale de Laiterie (Normes FIL-IDF $56: 1970$ ) (fig. 2). Toutefois, les pressions sont exprimées en centimètres de hauteur de colonne de mercure $(\mathrm{cm} \mathrm{Hg})$ et non en kilo-Newton par mètre carré $\left.\left(\mathrm{kN} / \mathrm{m}^{2}\right){ }^{1}\right)$.

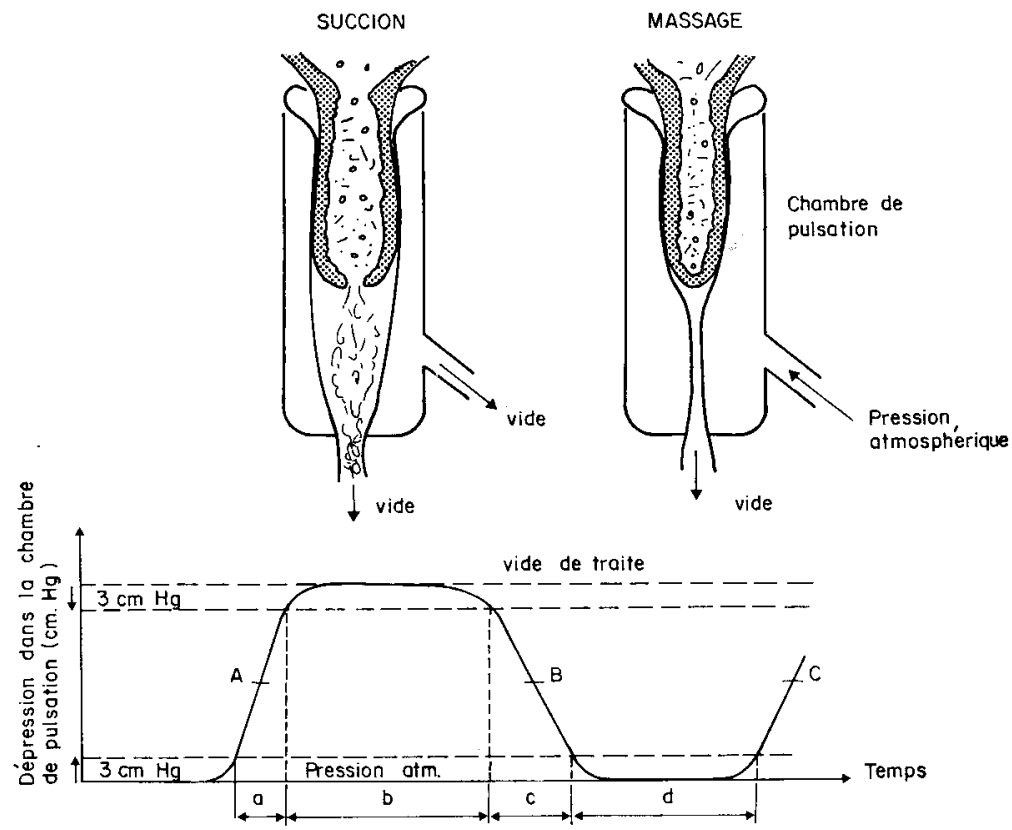

FIG. 2. - Définition de la pulsation

$a:$ application de la succion $\dot{o}:$ succion
$c:$ application du massage
$d:$ massage durée de chaque phase mesurée à $3 \mathrm{~cm}$ de $\mathrm{Hg}$ du maximum et minimum de la pression

Vitesse de pulsation (V) : nombre de cycles par minute.

Rapport de pulsation ( $R$ )

- anciennement $\mathrm{AB} / \mathrm{BC}$ (mesure à un niveau de dépression conventionnel)

- actuellement $\frac{a+b}{a+b+c+d} \times 100$ p. I00

\section{b) Envegistrement.}

La Chaîne de mesure, utilisée se compose :

- d'un enregistreur oscillographique à trace directe de marque Bell et Howell, référence 5 I27 p. 9-12, équipé de galvanomètres haute performance; référence 7342 , de bande passante o à $135 \mathrm{~Hz}$ (à \pm 5 p. IOO).

- de deux capteurs de pression à jauges, à fils extensiométriques tendus, de marque Bell et Howell - référence $4326 \mathrm{~L} 479$.

L'enregistrement de la pulsation est effectué au "niveau du gobelet", les capteurs étant montés conformément à la figure 3 .

La chaîne est étalonnée pour qu'un vide de $38 \mathrm{~cm}$ de mercure provoque un déplacement du " spot" de $76 \mathrm{~mm}$ et que la base de temps permette un lignage du papier de période o, $\mathrm{I}$ seconde à une vitesse de déroulement de $80 \mathrm{~mm} / \mathrm{s}$ (ceci autorise une précision de mesure des temps de o,Or seconde environ).

(1) $100 \mathrm{kN} / \mathrm{m}^{2}=\mathrm{I}$ bar $=\mathrm{I}, 02 \mathrm{kgf} / \mathrm{m}^{2}=75 \mathrm{~cm} \mathrm{Hg}$. 


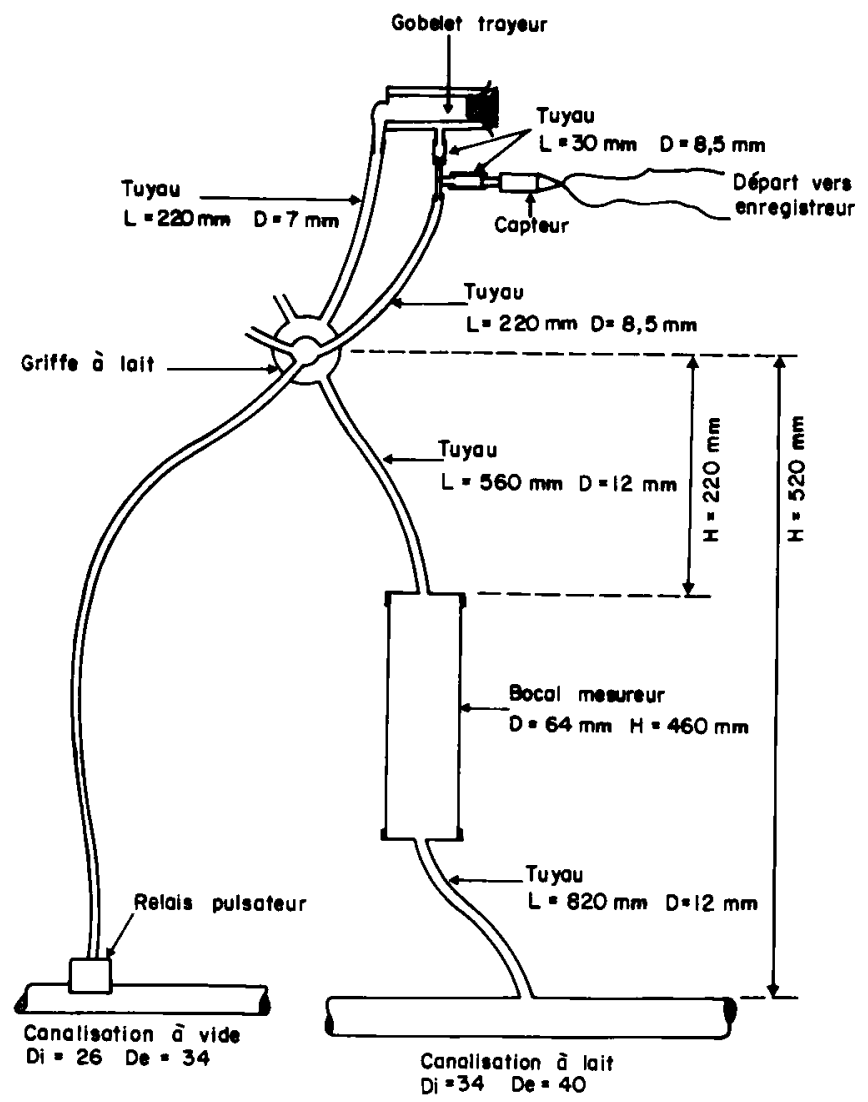

FIG. 3. - Schéma de branchement du capteur de pression au niveau du gobelet

\section{TABLEAU 3}

Valeurs mesurées du rapport $R$, de la vitesse $V$ et des phases $\mathrm{a}, \mathrm{b}, \mathrm{c}, \mathrm{d}$ de la courbe de pulsation pour chacune des combinaisons ( $\mathrm{R}, \mathrm{V})$ affichée $\mathrm{R}, a, b, c, d: \mathbf{I} 2$ données par combinaison (R, $\mathrm{V})$ $\mathrm{V}$ : ro données par combinaison $(\mathrm{R}, \mathrm{V})$

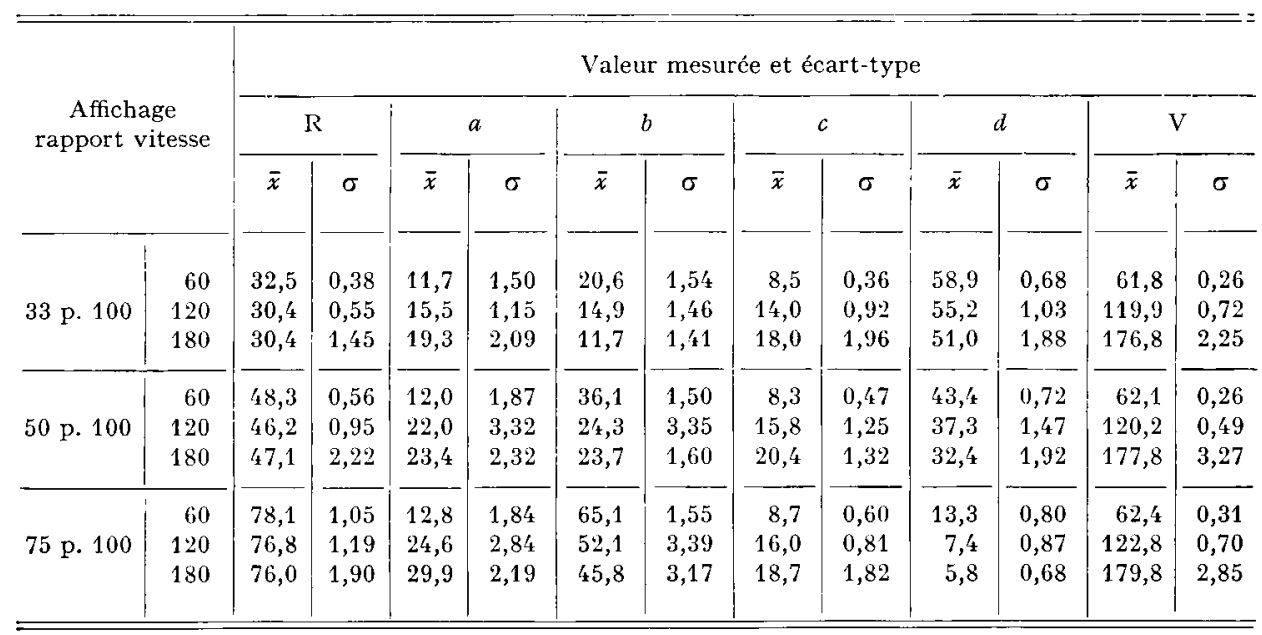


c) Caractéristiques mesurées.

Pour chaque combinaison, le rapport $(\mathrm{R})$ et les phases $a, b, c, d$, sont définies à partir de 2 enregistrements par poste - soit I2, au total - tandis que la vitesse est déterminée à partir de ro enregistrements répartis au hasard entre les 6 postes (1). Le tableau 3 et la figure 4 rassemblent les résultats des mesures effectuées.
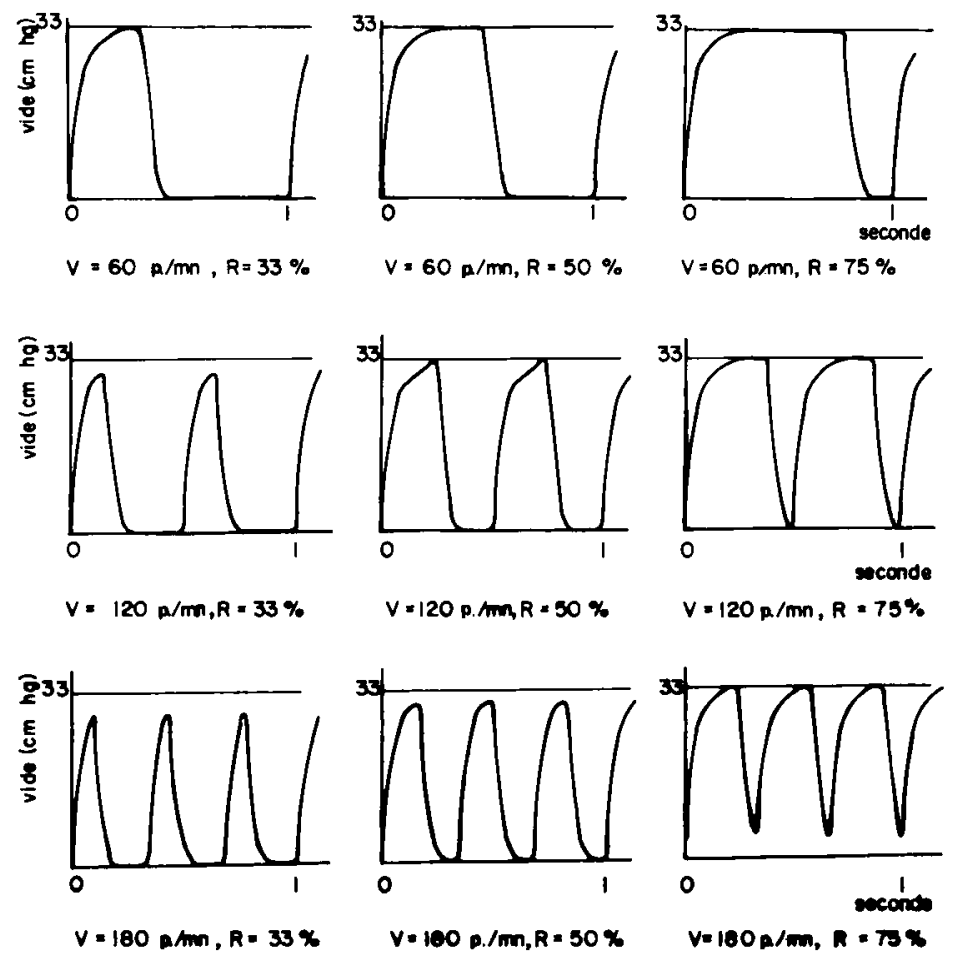

FIG. 4. - Aspects des enregistrements de courbes de pulsation obtenues pour chacune des combinaisons rapport-vitesse

(Enregistrements obtenus à la sortie de la chambre de pulsation : voir fig. 3)

D. - Critères zootechniques mesurés

I. Volumes de lait (en $\mathrm{ml}$ ).

La production laitière journalière, déduite de la mesure des 2 traites de 2 journées successives est caractérisée par :

- le lait machine réel (LMR) : volume de lait extrait par la machine sans l'intervention du trayeur; - le lait d'égouttage machine (EM) : volume de lait obtenu à la machine lors du massage pratiqué par le trayeur ;

- le lait d'égouttage manuel ou "repasse " (R) : volume de lait trait à la main après enlèvement des gobelets;

- le lait d'égouttage total (LET) : somme (EM + R);

- le lait total (LT) : volume total de lait recueilli, soit (LMR + LLT).

2. Quantité de matières grasses (MG en g) déduite de la mesure à chacune des 2 traites quotidiennes du taux butyreux (méthode Gerber) déterminé après mélange et homogénéisation du lait machine réel (LMR) et du lait d'égouttage total (LET).

(1) La "vitesse de pulsation " est la fréquence du signal de sortie du générateur de signaux (maître pulsateur). Il s'agit donc d'une caractéristique intrinsèque à celui-ci et semblable pour tous les postes. 
3. Descentes de lait.

La cinétique d'émission du lait est enregistrée pour chaque trayon toutes les 4 secondes au moyen de 2 suiveurs de niveau Sefram SN 25 et d'un potentiomètre Meci à 2 voies, type Speedomax (LABUSSiÈre et MARTiNet, 1964).

4. Périodicité des contrôles.

Les modalités selon lesquelles sont effectués les contrôles de matières grasses, de production laitière et de descente de lait sont rapportées à la figure 5 .
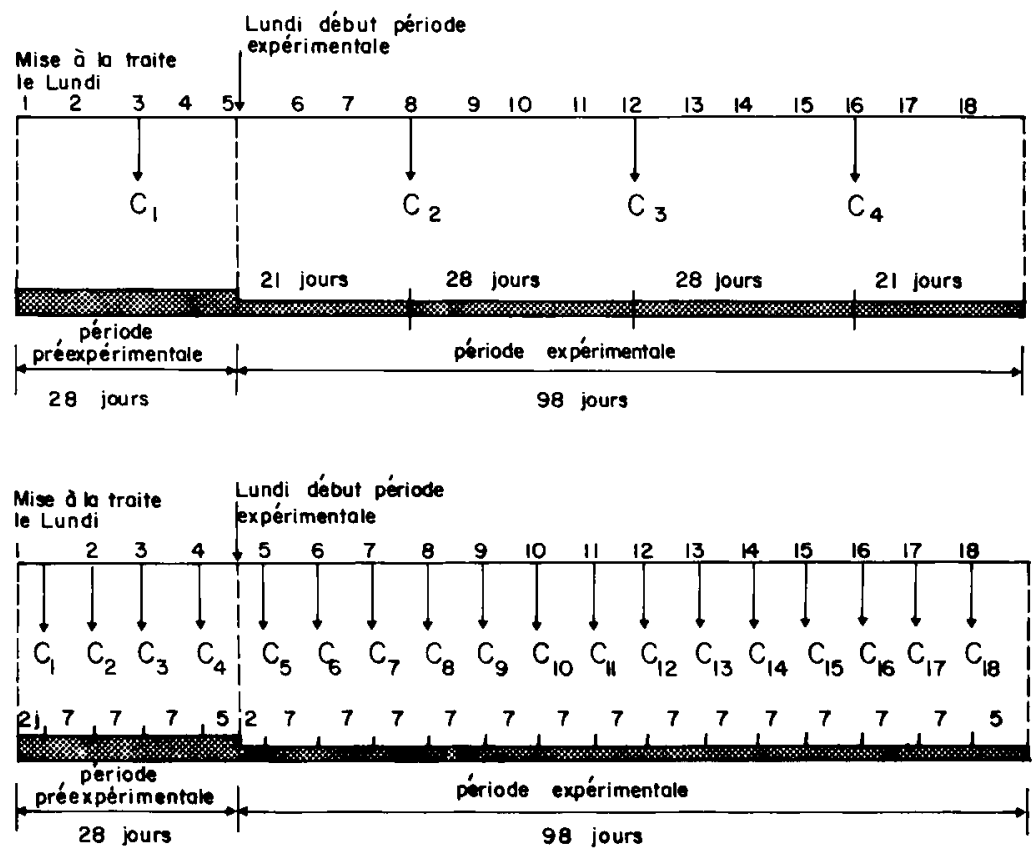

FIG. 5. - Périodicité des contrôles de matières grasses et de production laitière

- en haut : matières grasses : les contrôles $\mathrm{C}_{1}, \mathrm{C}_{2}, \mathrm{C}_{3}, \mathrm{C}_{4}$ ont lieu le lundi matin et le lundi soir.

- en bas : production laitière: Les contrôles $\mathrm{C}_{1}, \mathrm{C}_{2} \ldots \mathrm{C}_{17}, \mathrm{C}_{18}$ ont lieu le mardi et le mercredi, matin et soir.

Les contrôles de descente du lait sont effectués de la façon suivante:

- en période pré-expérimentale - aux 2 traites d'un des 6 jours de la $3^{\text {e }}$ semaine

- en période expérimentale -3 journées complètes $\left(4^{\mathrm{e}}, 7^{\mathrm{e}}\right.$ et $10^{\mathrm{e}}$ semaine $)$.

\section{Méthodes d'analyse statistique.}

Pour chacun des critères définis précédemment, les productions par brebis sont évaluées par la méthode Fleischman séparément pour la période pré-expérimentale et pour la période expérimentale, puis les performances globales obtenues par chacun des lots sont comparées entre elles par analyse de variance à une voie de classification.

En outre, pour la période expérimentale seulement, les effets des facteurs vitesse et rapport sont testés par analyse de variance selon le modèle mathématique :

$$
\mathrm{Y} i j k=m+a i+b j+c i j+e i j k
$$

où Yijk est la valeur du critère mesuré sur le $k^{\text {ien }}$ animal du lot soumis à une vitesse de pulsa-

tion $i$, et au rapport de pulsation $j$

$m$ : la valeur moyenne du critère

$a i:$ l'effet de la vitesse de pulsation $i$ 
$b j:$ l'effet du rapport de pulsation $j$

cij : l'interaction vitesse $\times$ rapport

eijk : l'erreur aléatoire propre à l'animal.

\section{RÉSULTATS}

\section{A. - EFFFETS DES 9 TYPeS DE PULSATIONS SUR LA PRODUCTION LATTIÈRE ET LES CARACTÉRISTIQUES DE TRAITE}

\section{I. - Évolution des productions journalières au cours de la lactation}

I1 apparaît à la figure 6 que la similitude des courbes de lactation observée au cours des 28 premiers jours pré-expérimentaux est conservée pendant les 98 jours de traite avec les 9 types de pulsation.

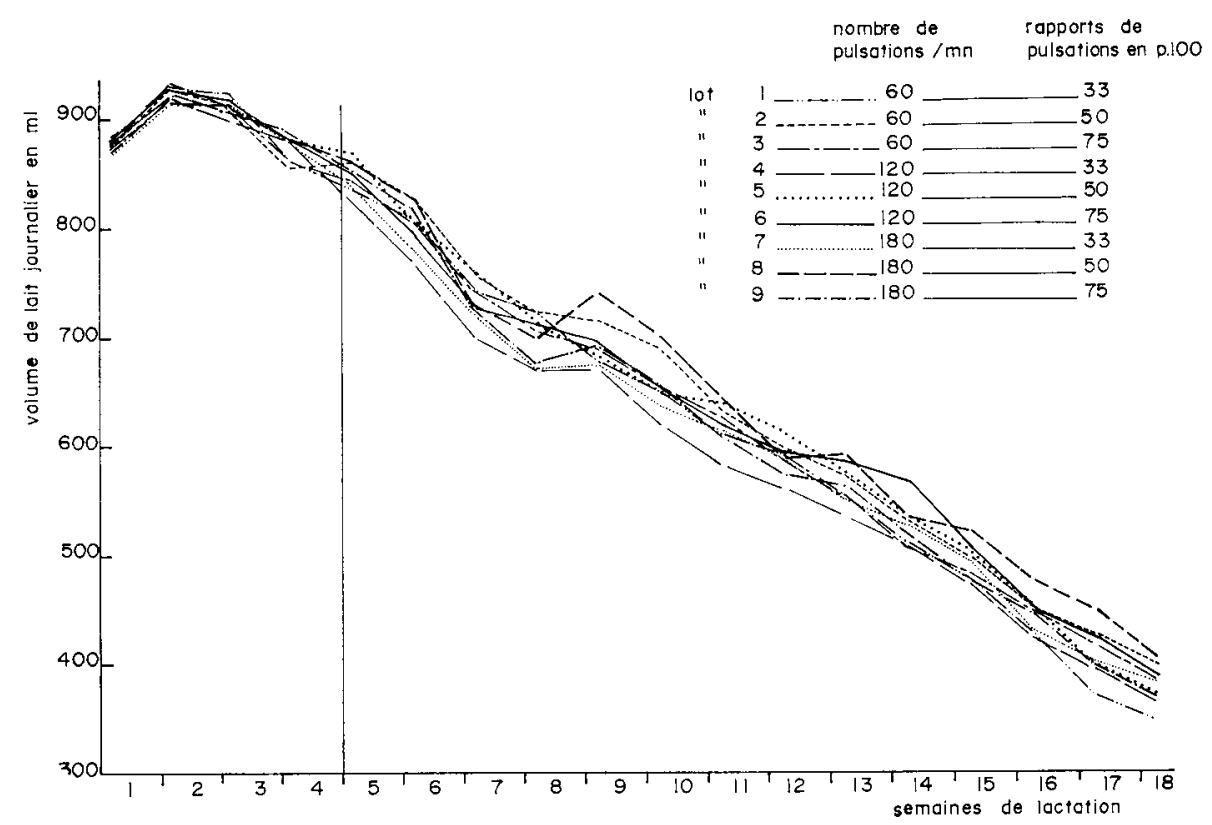

FIG. 6. - Courbes de production laitière des 9 groupes d'animaux au cours des périodes :

- pré-expérimentales (à gauche du trait vertical, 4 semaines)

- expérimentales (à droite du trait vertical, I4 semaines)

Par contre (fig. 7), les volumes d'égouttage (mantel: $\mathrm{R}$ et total LET) des lots 8 ( $80 \mathrm{p} / \mathrm{mn}, 50 \mathrm{p}$. I00) et 9 ( $180 \mathrm{p} / \mathrm{mn}, 75 \mathrm{p}$. IOO) se situent à un niveau nettement supérieur à celui des autres groupes pendant toute la période expérimentale.

On notera toutefois que ce phénomène peut résulter d'une erreur d'échantilonnage puisqu'il est déjà " amorcé " lors du dernier contrôle pré-expérimental. 


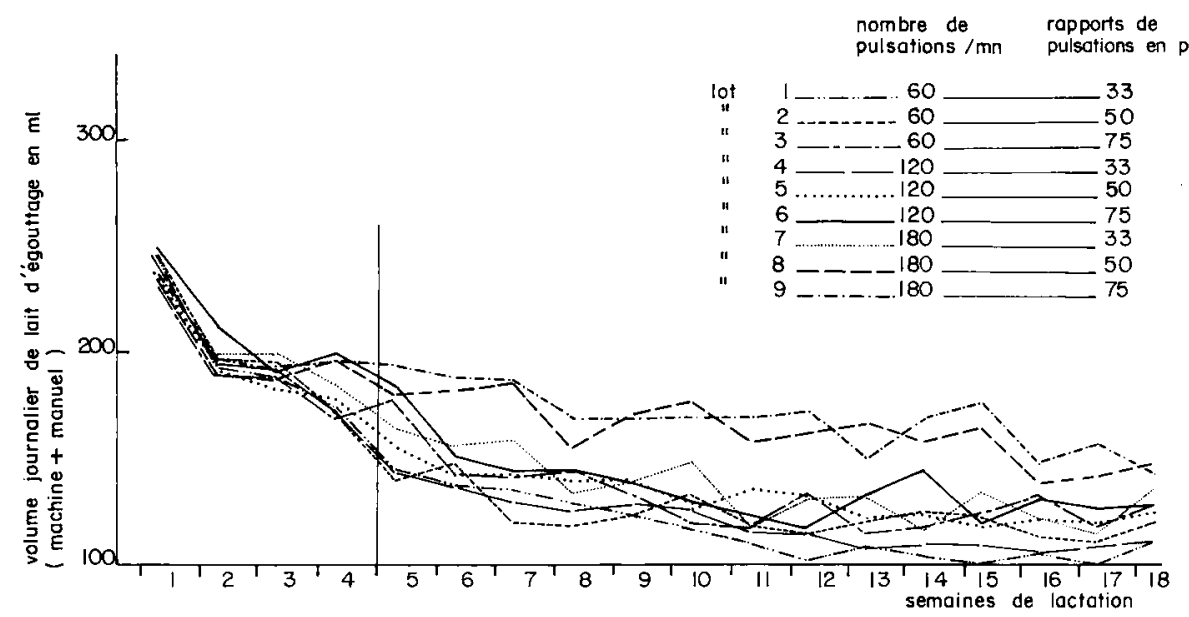

FIG. 7. - Évolution du lait total d'égouttage au cours des périodes :

- pré-expćrimentales (à gauche du trait vertical 4 semaines)

- expérimentales (à droite du trait vertical I4 semaines)

\section{2. - Productions totales recueillies au cours de chaque période}

La méthode de Fleichman appliquée à chaque animal conduit aux résultats présentés au tableau 4.

a) Pendant la période pré-expérimentale de 28 jours.

Les lots constitués a posteriori sont équilibrés car on ne relève entre eux aucune différence significative et ceci quelque soit le critère retenu.

A l'exception de la remarque mentionnée précédemment concernant une erreur possible d'échantillonnage, on doit donc admettre que les écarts qui pourraient se manifester par la suite, pendant la période expérimentale, seront provoqués par les traitements à l'essai. Néanmoins, les valeurs moyennes obtenues alors devront nécessairement présenter de grandes différences entre elles pour être significatives car la dispersion des données est relativement importante. Celle-ci est toutefois du même ordre pour tous les lots puisque les variances sont semblables.

b) Pendant la période expérimentale de 98 jours.

Aucune des 9 combinaisons n'influence :

- les volumes de lait total, de lait machine réel et d'égouttage machine ${ }^{1}$ ) ;

- la production de matières grasses.

Seuls l'égouttage manuel et l'égouttage total diffèrent significativement d'un lot à l'autre et c'est pourquoi nous n'avons approfondi l'analyse statistique que pour ces deux critères.

La comparaison entre traitements pris 2 à 2 est effectuée par le test de Duncan. Les résultats, que nous n'avons pas jugé utiles de rapporter en détail ici, sont schématisés en joignant d'un trait les lots entre lesquels ce test ne révèle pas de différences $\left({ }^{2}\right)$.

(1) La valeur de F pour ce critère n'est pas éloignée du seuil de signification.

( ${ }^{2}$ Seules les comparaisons " (I $80-75$ p. IOo)" " $(60-33$ p. IOo) ) pour l'égouttage machine et "( $80-75$ p. IOO) " "( 20 - 33 p. IOo) " pour l'égouttage manuel dépassent le seuil de signification de o,or. Les autres différences atteignent seulement le niveau o,o5. 


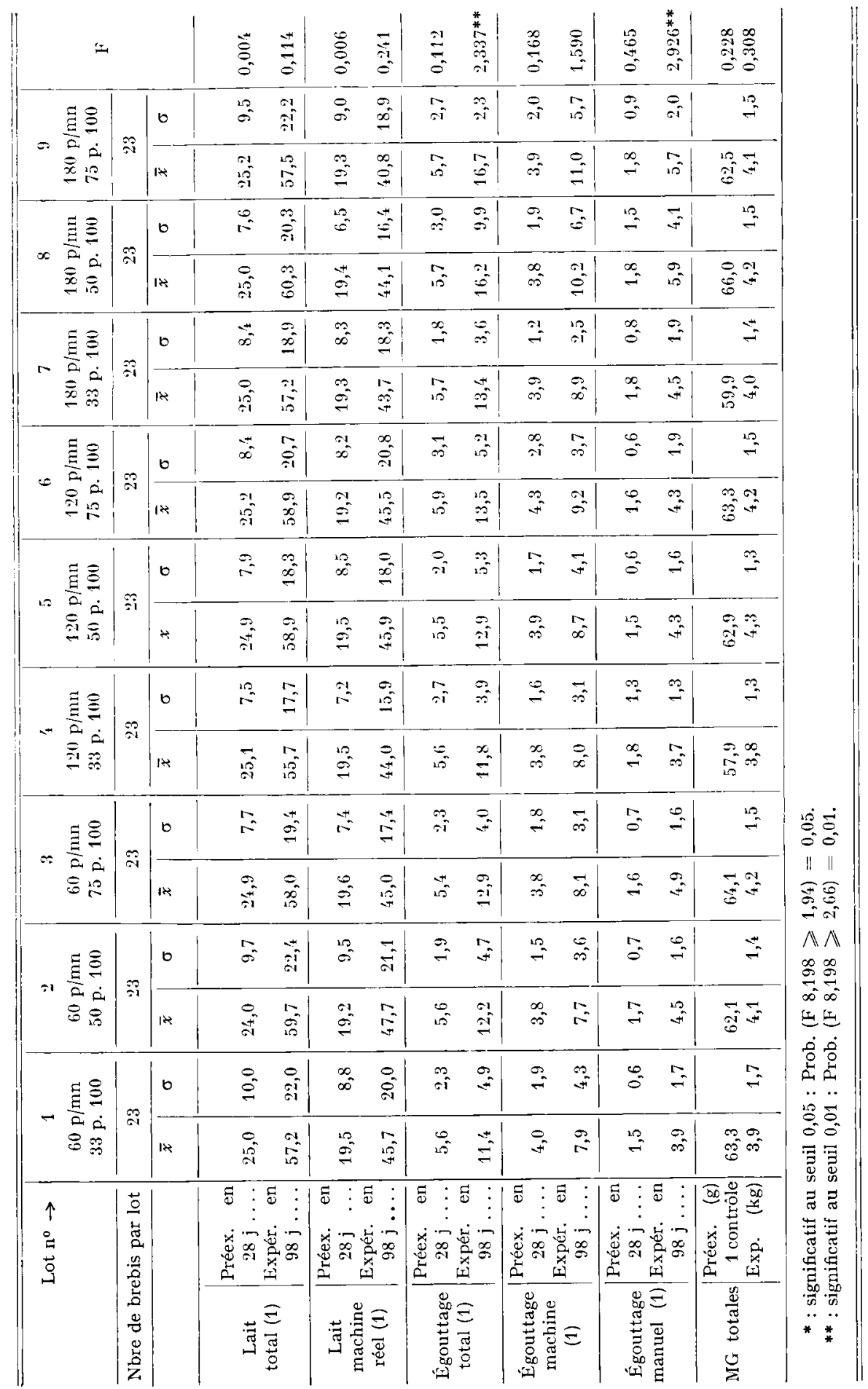


Égouttage total

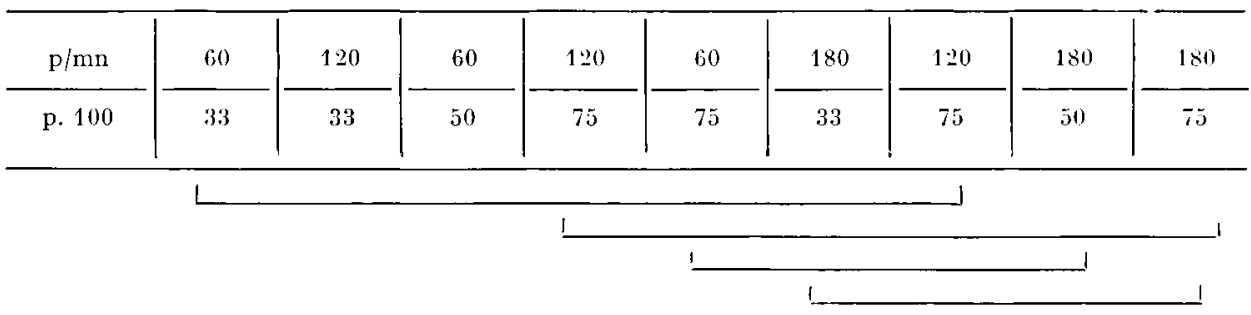

Égouttage manuel

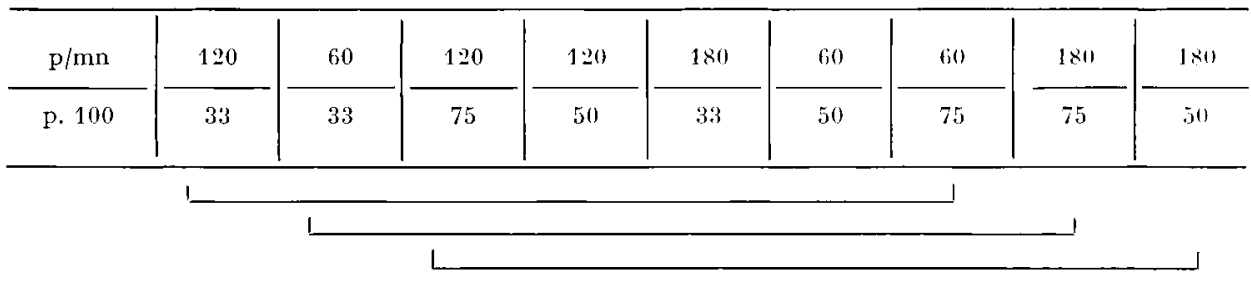

Il apparaît donc que la vitesse la plus rapide ( $180 \mathrm{p} / \mathrm{mn}$ ) associée à des rapports succion massage élevés (5o p. Ioo ou 75 p. Ioo) conduit à des volumes d'égouttage plus importants que ceux obtenus avec les combinaisons $(60-33 \mathrm{p}$. roo) et (I20-33 p. IOo) qui peuvent donc être considérées comme les plus "favorables".

\section{B. - INFLUENCE RESPECTIVE DE LA VITESSE E'T DU RAPPORT DE PULSATION}

L'étude précédente ne permet pas d'expliquer l'action séparée, ou l'interaction des deux paramètres de pulsation.

L'analyse de variance à 2 facteurs de classification que nous présentons ci-dessous répond à cette exigence.

Le tableau 5 prouve que, ni la vitesse, ni le rapport, n'affectent significativement les productions de lait total, de lait machine réel et de matières grasses. Ces résultats confirment ceux déjà obtenus précédemment puisque pour ces 3 critères nous arions conclu à l'identité d'effet des 9 combinaisons.

Par contre la lecture du tableau 5 révèle que:

- la vitesse a un effet significatif sur l'égouttage machine et hautement significatif sur l'égouttage manuel et total;

- le rapport succion massage n'affecte significativement que l'égouttage manuel. Comme il n'existe pas d'interaction entre les 2 paramètres de pulsation, on peut également conclure que pour chacune des 3 vitesses et pour chaque type d'égouttage, le classement relatif des 3 valeurs obtenues sera le même et ne dépendra pas du rapport envisagé.

Ce phénomène est particulièrement visible aux figures $8 d$ et $8 f$, bien qu'il ne soit pas exclu que, parmi les 3 rythmes de pulsation, deux d'entre eux puissent avoir une influence statistiquement identique. 


\section{TABLEAU 5}

Analyse statistique de l'infuence de la vitesse et du rapport de pulsation sur quelques critères de production

\begin{tabular}{|c|c|c|c|c|c|c|c|}
\hline \multirow{2}{*}{ Source de variation } & \multirow{2}{*}{$\begin{array}{c}\text { Degrés } \\
\text { de liberté }\end{array}$} & \multicolumn{6}{|c|}{ Carrés moyens et signification des tests $F$} \\
\hline & & Lait total & $\begin{array}{l}\text { Lait } \\
\text { machine }\end{array}$ & $\begin{array}{l}\text { Matieres } \\
\text { grasses }\end{array}$ & $\begin{array}{l}\text { Égouttage } \\
\text { machine }\end{array}$ & $\begin{array}{l}\text { Égouttage } \\
\text { manuel }\end{array}$ & $\begin{array}{c}\text { Égouttage } \\
\text { total }\end{array}$ \\
\hline Vitesse de pulsation & 2 & $4,948 \mathrm{NS}$ & $193,298 \mathrm{NS}$ & $0,055 \mathrm{NS}$ & $82,500^{*}$ & $30,781 * *$ & $206,057 * *$ \\
\hline Rapport de pulsation & 2 & $148,865 \mathrm{NS}$ & $80,478 \mathrm{NS}$ & $1,864 \mathrm{NS}$ & $22,274 \mathrm{NS}$ & $17,496 *$ & $85,144 \mathrm{NS}$ \\
\hline $\begin{array}{c}\text { Interaction } \\
\text { vitesse } \times \text { rapport }\end{array}$ & 4 & $17,334 \mathrm{NS}$ & $30,704 \mathrm{NS}$ & $0,339 \mathrm{NS}$ & $5,690 \mathrm{NS}$ & $2,012 \mathrm{NS}$ & $7,907 \mathrm{NS}$ \\
\hline Résiduelle & 198 & 411,599 & 347,283 & 2,108 & 18,261 & 4,468 & $32,8 \mathbf{4} 9$ \\
\hline
\end{tabular}

NS : effet non significatif.

* : effet significatif au seuil 0,05 .

$* *$ : effet significatif au seuil 0,01 .

\section{TALBEAU 6}

Différences entre valeurs moyennes obtenues pour chaque vitesse de pulsation (test de Duncan)

\begin{tabular}{|c|c|c|c|c|}
\hline & & $60 \mathrm{p} / \mathrm{mn}$ & $120 \mathrm{p} / \mathrm{mn}$ & $180 \mathrm{p} / \mathrm{mn}$ \\
\hline \multirow{2}{*}{$\begin{array}{l}\text { Égout tage } \\
\text { machine }\end{array}$} & moyenne (1) & 7,907 & 8,639 & 10,064 \\
\hline & $\begin{array}{r}60 \mathrm{p} / \mathrm{mn} \\
120 \mathrm{p} / \mathrm{mn}\end{array}$ & - & $\begin{array}{c}0,732 \mathrm{NS} \\
-\end{array}$ & $\begin{array}{l}2,157^{* *} \\
1,425^{*}\end{array}$ \\
\hline \multirow{2}{*}{$\begin{array}{l}\text { Égouttage } \\
\text { manuel }\end{array}$} & moyenne (l) & 4,333 & 4,090 & 5,380 \\
\hline & $\begin{array}{r}60 \mathrm{p} / \mathrm{mn} \\
120 \mathrm{p} / \mathrm{mn}\end{array}$ & - & $\underline{0,343 \mathrm{NS}}$ & $\begin{array}{l}0,947 * * \\
1,290 * *\end{array}$ \\
\hline \multirow{2}{*}{$\begin{array}{l}\text { Égouttage } \\
\text { total }\end{array}$} & moyenne (l) & 12,201 & 12,739 & 15,426 \\
\hline & $\begin{array}{r}60 \mathrm{p} / \mathrm{mn} \\
120 \mathrm{p} / \mathrm{mn}\end{array}$ & - & $\begin{array}{c}0,539 \mathrm{NS} \\
-\end{array}$ & $\begin{array}{l}3,226 * * \\
2,687 * *\end{array}$ \\
\hline
\end{tabular}

NS : différence non significative.

* : différence significative au seuil 0,05 .

** : différence significative au seuil 0,01 . 


\section{C. - Comparaison des 3 vitesses}

Pour réaliser cette étude nous avons associé les résultats des 3 lots soumis à la même vitesse (sans tenir compte du rapport qui leur est affecté) puis comparé les moyennes ainsi obtenues à l'aide du test de Duncan.

Cette analyse statistique dont les résultats sont présentés au tableau 6 conduit aux mêmes conclusions pour les 3 séries d'égouttage (machine, manuel et total): on ne relève pas de différence entre les effets des vitesses 60 et 120 alors que le rythme de $180 \mathrm{p} / \mathrm{mn}$ entraîne des quantités d'égouttage significativement plus importantes.

Cela revient à considérer les courbes des figures $8 b, 8 d$ et $8 f$, reliant les vitesses 60 et I20, comme étant confondues et elles-mêmes distinctes de la courbe obtenue avec I80 pulsations.

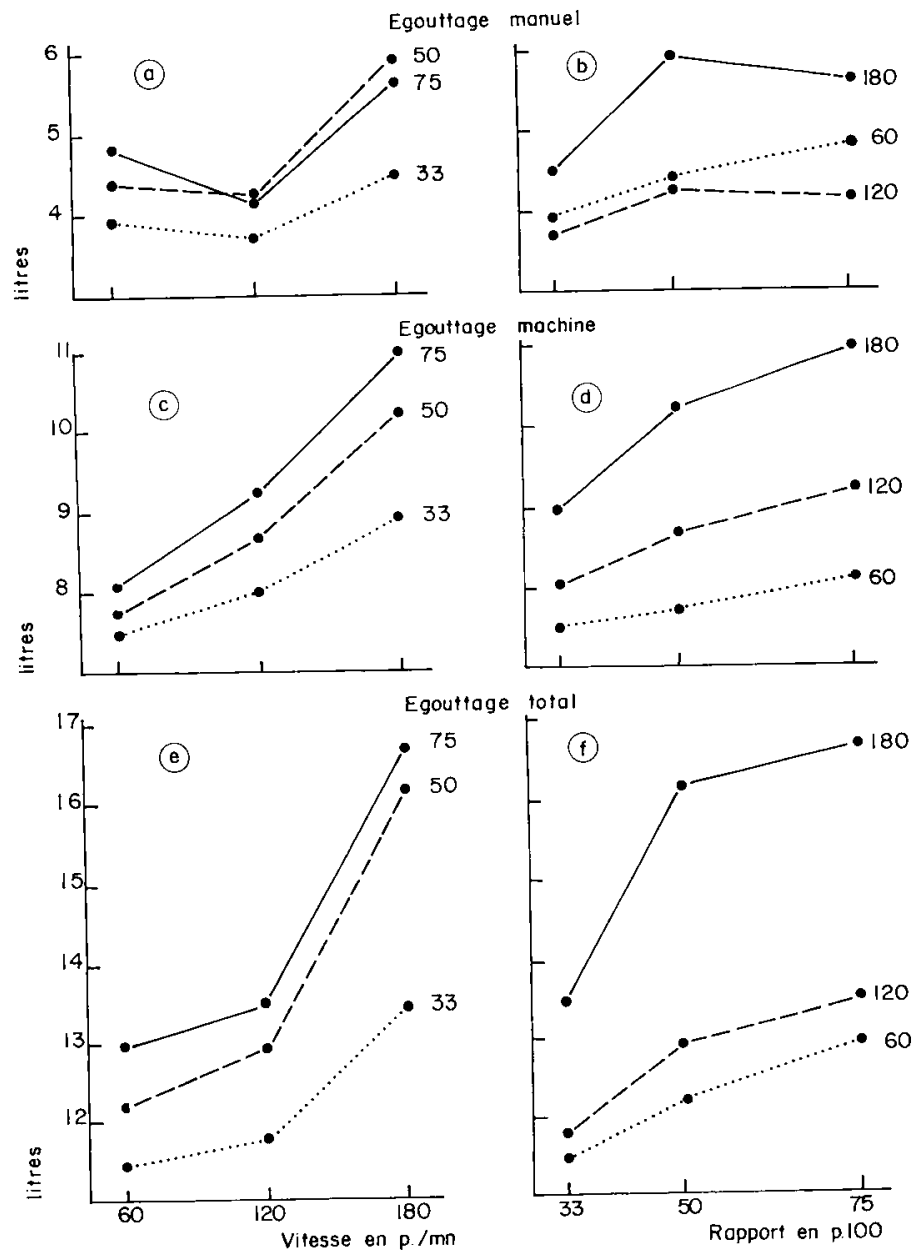

FIG. 8. - Influence du rapport et de la vitesse de pulsation sur le volume de lait obtenu lors des opérations d'égouttage chez la brebis Préalpes (volumes d'égouttages exprimés en litres par brebis en 98 jours)
(a) et (b) : égouttage manuel
(c) et $(d)$ : Egouttage machine
(e) et $(f)$ : égouttage total 


\section{D. - Comparaison DES 3 RAPPORTS (uniquement sur l'égouttage manuel).}

En procédant de la même façon que précédemment mais en négligeant cette fois le facteur vitesse nous sommes amenés à conclure que le rapport $33 \mathrm{p}$. Ioo réduit significativement le volume de repasse en comparaison des deux autres traitements (50 p. roo et 75 p. roo) qui ne semblent pas différer entre eux (tab1. 7 ).

Les 2 tracés de la figure $8 a$, les concernant peuvent donc être assimilés à une seule et même courbe.

\section{TABLEAU 7}

Différences entre valeurs moyennes obtenues pour chaque rapport de pulsation

(test de Duncan)

\begin{tabular}{|c|c|c|c|c|}
\hline \multirow{4}{*}{$\begin{array}{l}\text { Égouttage } \\
\text { manuel }\end{array}$} & Rapport & 33 p. 100 & 50 p. 100 & 75 p. 100 \\
\hline & Moyennes (l) & 4,053 & 4,911 & 4,938 \\
\hline & Rapport & & & \\
\hline & $\begin{array}{l}33 \text { p. } 100 \\
50 \text { p. } 100\end{array}$ & & $0,858^{*}$ & $\begin{array}{l}0,885^{*} \\
0,027 \text { NS }\end{array}$ \\
\hline
\end{tabular}

NS : différence non significative.

* : différence significative au seuil 0,05 .

E. - IMPORTANCE DES DIFFÉRENTES PHASES DE LA PULSATION

\section{I. - Infuence du "temps disponible " pour la fermeture du manchon}

Les effets bénéfiques de la réduction de la vitesse et du rapport de pulsation semblent résulter de l'augmentation du temps (1) dont dispose alors le manchon pour se fermer et masser efficacement le trayon. La figure 9 indique en effet que les volumes d'égouttage sont d'autant plus importants que ce temps est court. Cette constatation s'applique tout particulièrement à la combinaison $\mathrm{I} 8 \mathrm{o} \mathrm{p} / \mathrm{mn}-75 \mathrm{p}$. Ioo au cours de laquelle la pression atmosphérique n'a plus le temps de " s'installer " dans l'espace annulaire du gobelet (fig. 4).

\section{2. - Influence du "temps disponible " pour la succion}

De façon similaire, il apparaît que l'accroissement du temps disponible pour la succion se traduit (à vitesse constante) par une augmentation de l'égouttage qui pourrait s'expliquer par l'étranglement de la base du trayon consécutif à la péné-

(1) Temps représenté par $(c+d)$. 
tration excessive de celui-ci dans le manchon. On constate, par exemple, à $180 \mathrm{p} / \mathrm{mn}$ des volumes d'égouttage machine de 8,861 , ro,20 1 et Ir,o0 1 pour des temps de succion $(a+b)$ respectifs de 0, IO 7 s, 0,I59 s et $0,255 \mathrm{~s}$.
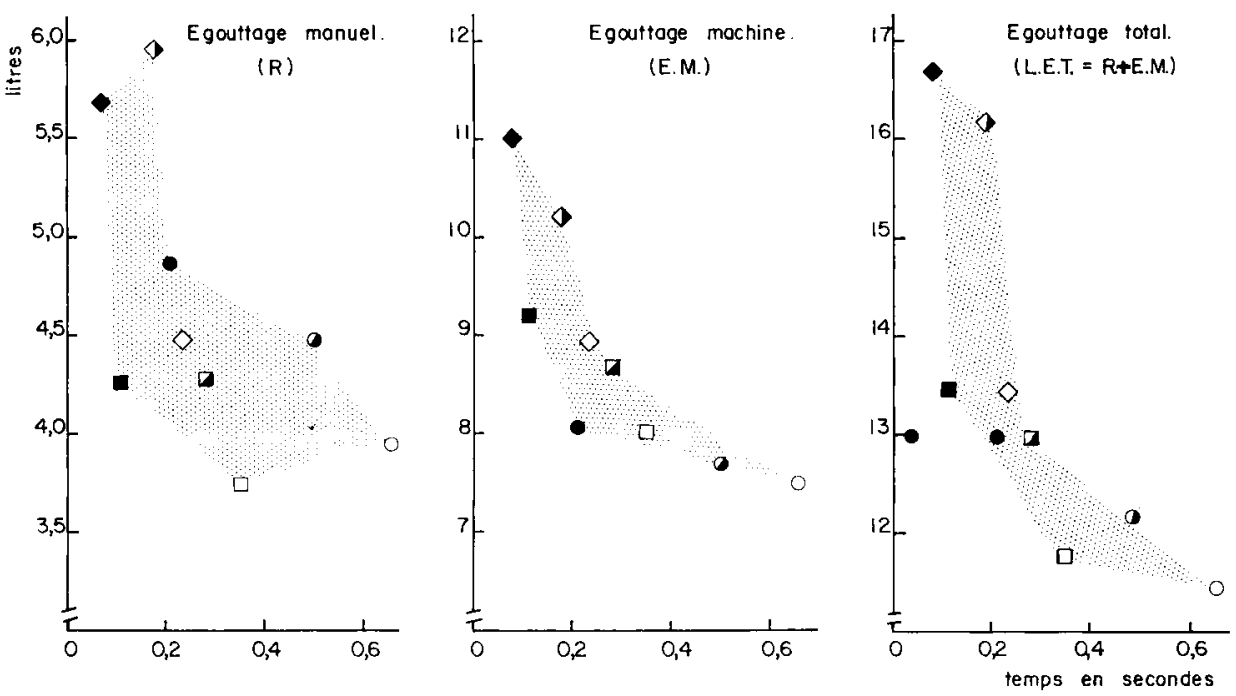

Fig. 9. - Relations entre le volume d'égouttage et le temps (t) disponible pour effectuer le massage.

$(\mathrm{R}, \mathrm{EM}$, et LET) sont exprimées en litres par brebis en 98 jours

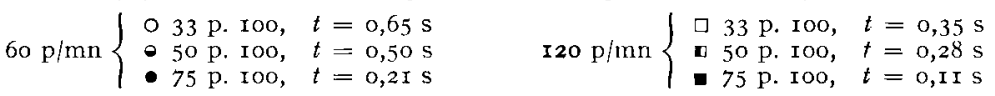

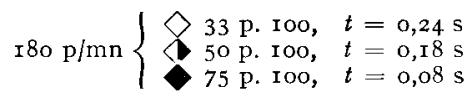

\section{3. - Infuence des différentes phases de la pulsation}

Compte tenu des caractéristiques du matériel utilisé pour produire la pulsation, il apparaît (fig. 4) qu'à rapport constant les phases transitoires $a$ et $c$, exprimées en pourcentage du cycle, décroissent avec la vitesse.

A titre d'exemple, l'égouttage machine diminue de $13,3 \mathrm{p}$. Ioo lorsque la phase (a) passe de 29,9 p. IOO à I 9,3 p. IOO (1).

Ces modifications pourraient également expliquer la réduction du lait d'égouttage.

\section{F. - RELATIONS ENTRE LES CARACTÉRISTIQUES DE LA PULSATION E'T LES COURBES DE DESCENTE DU LAIT}

Bien que le nombre d'animaux à 2 émissions ne soit pas tout à fait identique dans les 9 lots pré-expérimentaux, il semble que ce type de brebis a tendance à disparaitre plus rapidement au cours de la période expérimentale avec les traitements 7,8 et 9 faisant intervenir le rythme de pulsation le plus rapide.

(1) Ces valeurs sont calculées à I $80 \mathrm{p} / \mathrm{mn}$ pour les rapports respectifs de $75 \mathrm{p}$. Ioo et $33 \mathrm{p}$. Ioo. 
Le regroupement des lots soumis à la même vitesse ou au même rapport (tabl. 8)

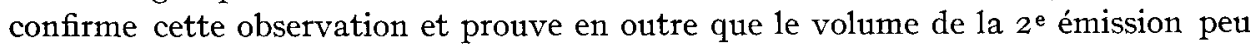
affecté par le changement du rapport succion massage, est sensiblement réduit lorsque l'on accroît la vitesse

\section{TABLEAU 8}

Influence du rapport et de la vitesse de pulsation sur quelques caractéristiques de descente du lait

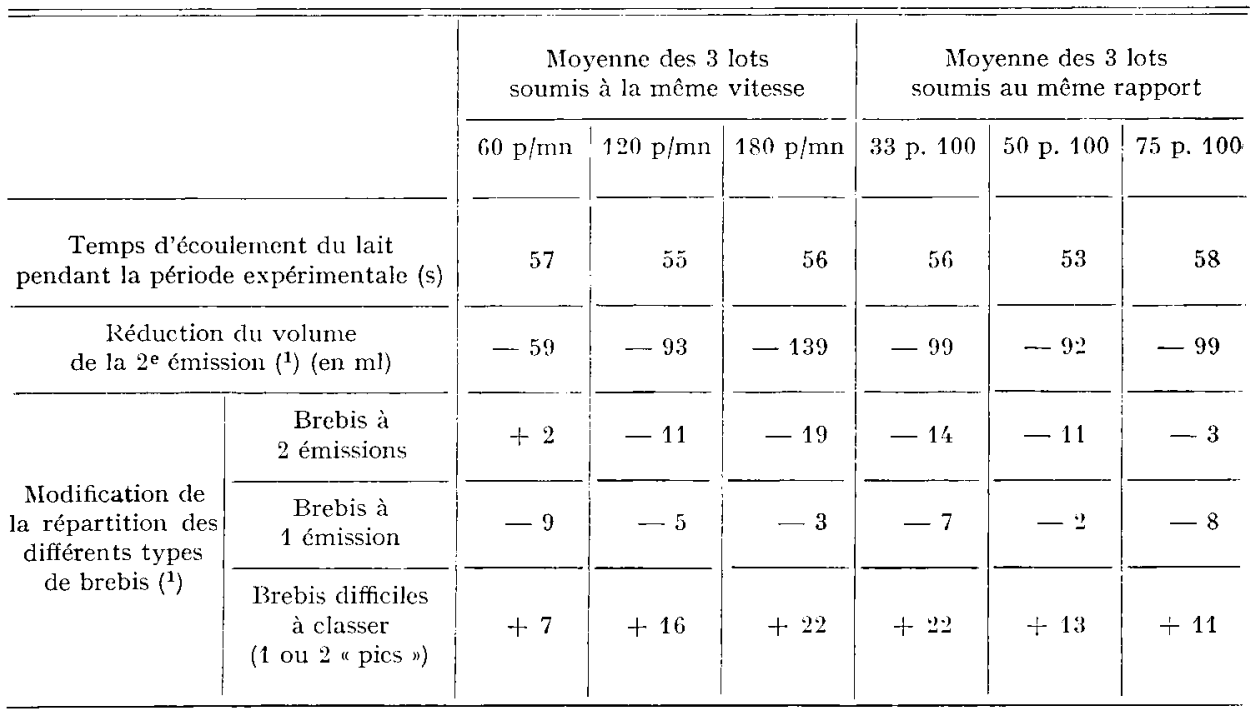

(1) Ces modifications représentent les différences entre la période expérimentale et la période préexpérimentale.

Les volumes d'égouttage importants recueillis à 180 pulsations pourraient ainsi partiellement résulter d'une inhibition partielle ou total du réflexe d'éjection du lait.

On remarquera enfin que les changements des paramètres de pulsation ont relativement peu d'influence sur le temps d'écoulement du lait. Il en résulte que le moment à partir duquel le trayeur peut commencer à pratiquer l'égouttage ne doit pas être modifié.

\section{DISCUSSION ET CONCLUSION}

A l'issue de ce travail nous pouvons conclure qu'il est possible (sinon recommandé) de traire les brebis avec d'autres paramètres de pulsation que ceux traditionnellement utilisés jusqu'à maintenant ( $180 \mathrm{p} / \mathrm{mn}, 50$ p. IOO). 
En effet les 9 groupes de 23 brebis (préalablement équilibrés après une période pré-expérimentale de 28 jours) fournissent en 98 jours la même production totale de lait et de matières grasses.

Toutefois les volumes recueillis lors des interventions d'égouttage sont significativement plus faibles avec des combinaisons faisant intervenir les vitesses les plus lentes $(60 \mathrm{p} / \mathrm{mn}$ et $\mathrm{I} 20 \mathrm{p} / \mathrm{mn}$ ) et le rapport le plus bas (33 p. Iоo).

Ceci est confirmé par l'analyse de variance à 2 facteurs de variation qui complète la comparaison globale des 9 traitements et qui révèle que la diminution de la vitesse affecte davantage l'égouttage que la réduction du rapport.

Cette constatation est particulièrement cohérente si l'on sait que, dans les 2 cas, (et tout particulièrement dans le premier) il en résulte une augmentation du temps disponible pour la fermeture complète du manchon.

Les risques d'étranglement du trayon consécutif à son aspiration dans le manchon sont alors beaucoup plus faibles et ceci pourrait déjà suffire à justifier la réduction du lait d'égouttage. Sans négliger cette explication d'ordre mécanique proposée par MEIN (I973) dans une autre espèce et sans exclure le rôle des phases intermédiaires déjà signalées par PHILLIPS (I963) nous retiendrons surtout 1'hypothèse qui nous avait guidée au moment de la mise en place de cette expérience et qui concerne une meilleure efficacité de la phase de massage à 60 pulsations/mn.

Nous pensons, en effet, que la forte congestion des tissus mammaires constatée à $\mathrm{I} 8 \mathrm{o}$ pulsations risque de provoquer une rétention importante de lait dans les alvéoles à la suite d'une inhibition du réflexe neuro-endocrinien d'éjection.

Nous arons pu vérifier au cours de cette étude que de tels phénomènes d'inhibition (concrétisés par la disparition de la $2^{\mathrm{e}}$ émission et $1^{\prime}$ accroissement corrélatif du volume de l'égouttage) sont beaucoup plus fréquents avec les combinaisons de pulsations pour lesquelles les possibilités de "massage " sont insuffisantes (lots 7 , 8 et 9). Ce point capital avait d'ailleurs déjà été souligné par FLEURY qui écrivait en I933 : “Pour que les tissus soient à même de supporter sans fatigue et sans douleur l'action de la machine à traire, il faut et il suffit que le temps de repos (mas sage) soit le double du temps de travail (succion)".

Pour les mêmes raisons Drmov et al. (I965) attirent également l'attention sur la nécessité de préserver un temps suffisant pour que le manchon se ferme complètement mais les limites au-delà desquelles ces exigences ne sont plus remplies sont beaucoup moins rigoureuses que celles que nous pourrions être amenés à définir. C'est ainsi qu'à la suite d'une série d'études impliquant la modification successive :

- de la vitesse entre 50 et 200 pulsations au rapport imposé de 50 p. Ioo;

- du rapport entre $25 \mathrm{p}$. IoO et $75 \mathrm{p}$. Ioo à la vitesse imposée de $150 \mathrm{p} / \mathrm{mn}$; les chercheurs Bulgares considèrent comme optimum la combinaison $\mathrm{I}_{50} \mathrm{p} / \mathrm{mn}$ 66 p. Ioo qui offre l'avantage d'obtenir rapidement le maximum de lait sans bleuissement des trayons. Ces conditions sont évidemment très différentes de nos recommandations ( $60-33 \mathrm{p}$. Ioo) et de nos interprétations puisque ces auteurs estiment finalement que les changements du rapport de pulsation influenceraient davantage la qualité de la traite que les variations de la vitesse. Les effets de ce dernier paramètre sont toutefois très difficiles à appréhender dans l'expérience de Drmov car ils semblent dépendre à la fois du type de manchon et du critère mesuré.

Ainsi, pour le "temps de traite " le faisceau trayeur Alfa-Laval (que nous avons également utilisé) permet de donner successivement la préférence aux vitesses 80 , 
$50, \mathrm{I} 50,200$ et $\mathrm{I} 20 \mathrm{p} / \mathrm{mn}$. Il est regrettable de ce fait que la taille des lots, la race des brebis et la durée de l'essai n'aient pas été précisés car cela aurait peut-être permis d'expliquer les divergences existant avec notre propre travail.

L'examen des 3 publications récentes de Succi, Cutry et Enne (I970) ; Succi, ENNE et CUTRY (197I) et ENNE (1972) ne permet malheureusement pas au lecteur de se faire une opinion claire de ce sujet. Le faible nombre d'animaux par groupes (entre 3 et 6 ) et les changements fréquents dans le protocole expérimental conduisent en effet à des résultats contradictoires à partir desquels il semble se dégager une légète supériorité de la pulsation I5O-2/I. Soulignons, malgré tout, qu'après leur premier essai les auteurs italiens n'ont pas pu faire de distinction entre les effets des traitements $180-I / I$ et $60-3 / I$, ce qui concorde partiellement avec ce que nous avions nous-mêmes observé avec les lots 8 et 9 .

Ces quelques constatations sont également assez voisines de celles de KoraA (I966) qui ne trouve pas de différence entre les rapports I/I et 2/I) et de KRAmorov, MAR'TYNOV et YABLOCHKIN (I965) qui utilisent préférentiellement des rythmes de pulsation (70 à roo) finalement peu éloignés de ceux qui nous paraissent être les plus favorables.

Compte tenu de toutes ces considérations et avant de vulgariser l'emploi de la combinaison $60-33$ p. Ioo, il nous a paru justifié d'en confirmer l'efficacité avec des manchons trayeurs d'élasticité différente puisqu'il est admis que la qualité du massage est très dépendante de ce dernier paramètre.

Tout en réduisant sensiblement l'égouttage, la vitesse 60 permettrait de se dispenser de l'électricité (et des pulsateurs électromagnétiques nécessaires à $\mathbf{1} 80$ ) grâce à l'adoption de pulsateurs pneumatiques qui faciliteraient l'implantation de la traite mécanique des brebis dans certaines régions du bassin méditerranéen (montagnes par exemple...).

Reçu pour publication en mars 1974 .

\section{REMERCIEMENTS}

Cette étude a pu être entreprise grâce à l'aide de l'I. N. R. A., du Fond d'Orientation et de Régularisation des Machines agricoles et de la Société Alfa-Laval que nous tenons ici à remercier.

Nous exprimons également notre reconnaissance à l'Institut Technique de l'ilevage Bovin qui a bien voulu effectuer une partie du testage de la machine à traire et à l'ensemble du personnel de la ferme expérimentale de Brouessy dont la collaboration nous a été précieuse lors de la réalisation de ce travail.

\section{SUMMARY}

EFFECTS OF PULSATION RATE AND RATIO ON THE CHARACTERISTICS OF MECHANICAL MILKING IN PREALPES DU SUD EWES

The purpose of the present study was to compare the effects of 3 pulsation rates (6o, I 20 and I $80 \mathrm{p} / \mathrm{min})$ and of 3 suction-massage ratios $(33,50$ and $70 \mathrm{p}$. roo) on the milk yield and milking characteristics of Préalpes du Sud ewes. 
The general organization of the experiments is schematized in Fig. 6. During a pre-experimental period of 28 days, preceded by a short period of suckling ( 48 hours), all animals were adjusted to $50 \mathrm{p}$. I $100-\mathrm{I} 80 \mathrm{p} / \mathrm{mn}$.

After these first four weeks, the 207 ewes (the characteristics of which are reported in table I) were distributed into 9 balanced groups. During an experimental period of 98 days, each of these groups was subjected to one of the 9 treatments combining the 3 pulsation rates and 3 pulsation

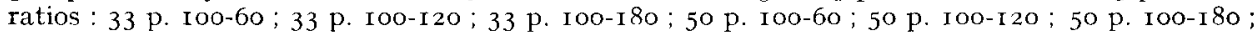
75 p. I00-60; 75 p. IOO-I $20 ; 75$ p. IOO-I 80 (fig. 4 ).

Table 4 shows that:

- These 9 combinations do not seem to affect either the production of fat or the total volume of milk, or the volume of milk extracted by the machine before the intervention of the milker or the volume of mechanical stripping.

- Manual stripping and total stripping significantly differ from one group to another and the Duncan test (tables 8 and 9) reveals that the highest pulsation rate $(\mathrm{I} 8 \mathrm{o} \mathrm{p} / \mathrm{mn}$.) associated with high suction-massage ratios (50 or 75 p. IOo) leads, for these 2 criteria, to larger volumes than those obtained with the associations $33 \mathrm{p}$. Ioo-6o and $33 \mathrm{P}$. IOo-I 20 , which consequently can be considered as the most favourable ones.

The analysis of variance presented in table 5 does not allow to detect interactions between the pulsation rate and ratio.

In contrast :

- Reduction of the pulsation rate entails a significant decrease in machine stripping and highly significant decrease in manual and total stripping (table 5).

- No statistical difference is observed between $60 \mathrm{p} / \mathrm{mn}$ and $\mathrm{I} 20 \mathrm{p} / \mathrm{mn}$, whereas $180 \mathrm{p} / \mathrm{mn}$ results in a definitely greater stripping (table 6).

- Modifications of the ratio only affect the manual stripping $(p=0.05$; table 5$)$. The stripping volume obtained at a ratio of $33 \mathrm{p}$. I oo is significantly lower than the volume collected with the treatments 50 and 75 p. Ioo, between which there does not seem to be any difference (table 7 ).

The favourable effect of reducing the pulsation rate and ratio seems to arise from the increase in the time available for the teat-cup to close and to efficiently decongest the teat (fig. 9).

\section{RÉFÉRENCES BIBLIOGRAPHIQUES}

Bosc J., I963. Problèmes de l'organisation du travail dans un troupeau de brebis laitières. Adaptation de la méthode arête de poisson à la traite mécanique des brebis. Compte vendu à l'A. F. Z., 3-39.

Bosc J., I972. The induction of synchronization of lambing with the aid of dexamethasone. J. Reprod. Fert., 28, 347-357.

Casu S., r967. La pecora sarda e la mungitura mecanica. Riv. Zootec (I), 32-48.

Dimov S., Ig63. Étude sur les possibilités de traite mécanique chez les brebis (en Bulgare). Bull. Inst. Mechaniz. Electrif. Agric., 4, 83-96.

Dimov S., Goraxor I., Garanitcheva K., Dobrev D., I965. Étude des possibilités pour une mécanisation des processus de travail dans l'élevage des brebis (en Bulgare). Economics and Mechanization of A griculture, 2 (5), 423-442.

Dimov S., TAxev I., Shalichev Y., Ig66. Mechanical milking of ewes. Mekhaniz Electrif Sel Stop 16 (4), 22-24 (en Bulgare) (in Dairy Sci. abstr., I968, 30 (8) 2 583).

ENNE G., I972. Prove di mungitura meccanica di pecore di razza Sarda-Ferzo ciclo di Sperimentazione. Il Latte, 46 (I), 3-I 4 .

FINcI M., 1957. The improvement of the Awassi breed of sheep in Israel. Bull. Res. Coun. Israel. 6 B. (in Dairy Sci. abstr., 1958, 20, 755).

Fueury R., I932. Cité par L. Galtier, I943. La traite des brebis. Thèse de médecine vétérinaire, E. N. V., Lyon.

GAAL M., 1968. Wachine millking studies with Hungarian combing wool merino ewes. Allattenyesztes, 17 (4) $325-37$.

Galtier L., I943. La traite des brebis. Leur lait et le fromage de Roquefort. Thèse de médecine vétérinaire (E. N. V., Lyon). Faculté de Medecine et Pharmacie de Lyon, I67 pages.

Horak F., 1964. Milk yield of sheep and the possibility of the use of machine milking. Sb. vys. Sk. Zemèd. Brno, (I) I $2 \mathrm{I}-33$. 
KolAR K., I966. The first results obtained with the experimental machine milking of sheep in czechoslovakia. Zemedelska technika, 12, (39), 73-96.

Kramorov YU. I., Martynov V. P., Yablochkin A. M., I965. Installation for the machine milking of ewes. Ovtsevodstvo, 11 (2), 32-33 (in Dairy Sci. Abst., 28, 1966).

Labussière J., Martinet J., ig64. Description de deux appareils permettant le contrôle automatique des débits de lait au cours de la traite à la machine. Premiers résul tats obtenus chez la brebis. Ann. Zootech., 13, 199-212.

LAbUSSIL̀RE J., 1966. Relations entre le niveau de production laitière des brebis et leur aptitude à la traite. XVII Congrès. Int. Lait. Munich. Section $A_{1}, 43^{-5} \mathrm{I}$.

LABUSSiERe J., I969. Importance, composition et signification des différentes fractions de lait obtenues successivement au cours de la traite mécanique des brebis. Ann. Zootech., 18, I85-I95.

Labussière J., Martinft J., Denamur R., ig68. The influence of the milk ejection reflex on the flow rate during the milking of ewes. J. Dairy Res, 36, I9I-20I.

Labussitre J., Ricordeau, G., ig7o. Aptitude à la traite mécanique des brebis de race Préalpes du Sud et croisées Frisons $\times$ Préalpes. Etude à différents stades de la lactation. Ann. Zootech., 19, I59-I9o.

Labussitire J., Combaud J.-F., Petreouin P., I974. Effets de la suppression de la traite du dimanche soir chez les brebis de race Préalpes du Sud. Ann. Zootech., 23, 435-444.

MASAR M., I968 a. Labour requirements at the first machine-milking of sheep in czechoslovakia as compared with the manual milking of sheep (en Tchèque). Vedecke Prace Vyskumneho vistavu ovciarskeko I Trencine, 4, 57-7 I.

MAsar M., I $968 \mathrm{~b}$. A study on various methods of labour or xanization at the first experimental machine milking of sheep mezechoslovakia. (en T'chèque). I'idecke Prace I'yskumneho vistavu ovciarskeko V Trencine, 4, 75-87.

Masar M., I $68 \mathrm{c}$. A study of labour requirement at machine milking done with the model of the czechoslovak milking machine and with the Alfa-Matic milking machine. Fedecke Prace I'yskumneho vistavn ovciarskeko V Trencine, 4, 9I-106.

MASAR M., I $968 d$. Time study on the milking of one sheep with the milking automaton Alfa-Matic and with the model of the czechoslovak milking automaton. Vedechke Prace Vyskumneho vistavu ovciarskeho I trencine, 4, Iog-120.

Masar MI., I972. Étude de la duréc de la traite mécanique d'une brebis moyennant les machines à traire automatiques D 20-8, D 20-I 6 de fabrication Tchécoslovaque et la machine automatique Alfa-Matic dans la salle de traite type Casse. I'edecke Prace I'uskumncho vistavu ovciarskcko I Trencine, 6, 53-59.

Mein G. A., Thiel C., Clovgh P. A., I973. The patterns of milk flow rate and teat movement in the test-cup liner during milking. Aust. J. Dairy. Tech. (sous presse).

Phillips D. S. M., 1963. The effect of pulsator ratio on milking rate. Proc. of Ruakura Farmers Conference $\mathrm{W}$ Veek, $\mathrm{I}-\mathrm{I} 5$.

Succi G., Cutry A., Enne (r., I97o. Primi risultati sulla mungitura meccanica delle pecore. Atti del $V$ Simposis Internationale di Zootechnia Milano, I68- I78.

Succi (i., linne G., Cutry A., r97I. Prove di mungitura meccanica di pecore di razza Sarda Risultati del secondo cido di Sperimentatione. Il Latte, 45 (3), 3-15.

Toulouse Combes A., I923. Directions pratiques pour l'élevage et l'exploitation des brebis laitières dans la région de Roquefort. Cité par Galtier, 1943, dans la Traite des Brebis. Thèse de médecine vétérinaire, E. N. V. de Lyon. 